\title{
Exposure to Alternating Hypoxia and Hyperoxia Causes Severe Proliferative Retinopathy in the Newborn Rat
}

\author{
JOHN S. PENN, MARK M. HENRY, AND BARBARA L. TOLMAN \\ Arkansas Center for Eye Research, University of Arkansas for Medical Sciences, \\ Little Rock, Arkansas 72205-7199
}

\begin{abstract}
ABS
Exposure to variable hyperoxia has recently been
shown to be much more effective at producing proliferative shown to be much more effective at producing proliferative hyperoxia. To incorporate a more clinically relevant oxygen-exposure paradigm in our studies, we have now used a cycle between 50 and $10 \%$ oxygen and have compared its effects with those found using new exposures to the previously used $80 / 40 \%$ cycle. Starting at birth and continuing for $14 \mathrm{~d}$, rats were exposed to environments that cycled between 50 and $10 \%$ oxygen or 80 and $40 \%$ oxygen every $24 \mathrm{~h}$. After exposure, some rats were killed for assessment of retinal vascular development. Others were removed to room air for $4 \mathrm{~d}$ before killing and evaluation for the presence of abnormal neovascularization-a clinical consequence believed to be promoted by termination of oxygen therapy. The 50/10\% cycle resulted in greater retardation of retinal blood vessel development during oxygen than that found in the $80 / 40 \%$ exposure group. After $4 \mathrm{~d}$
\end{abstract}

postexposure in room air, the incidence of preretinal neovascularization was $97 \%$ in the $50 / 10 \%$ rats and $72 \%$ in the $80 / 40 \%$ group. Clearly, the overall amount of oxygen the subject receives is less critical than other parameters of its administration in producing proliferative retinopathy. Also, the range of variation ( $40 \%$ in both cases) is not the controlling characteristic. Our results suggest that consistency of oxygen level and avoidance of hypoxic levels should be important concerns in neonatal oxygen therapy. (Pediatr Res 36: 724-731, 1994)

Abbreviations
$\mathbf{R O P}$, retinopathy of prematurity
$\mathrm{FiO}_{2}$, fractional inspired oxygen
$\mathrm{PaO}_{2}$, arterial blood oxygen partial pressure
$\mathbf{P a C O}_{2}$, arterial blood carbon dioxide partial pressure
ADPase, adenosine diphosphatase
ICU, intensive care unit

ROP is a complex disease involving multiple factors. Oxygen was first recognized as a critical factor in the early 1950s (1-3), but the potential for prolonged hyperoxia alone to cause or exacerbate ROP is still not clearly defined. The disease persists despite the attention now placed on careful monitoring and limited oxygen delivery. Additional doubt about the role of hyperoxia in the pathogenesis of ROP has been raised with the finding that cyanotic premature infants can develop ROP in the absence of oxygen therapy (4). The theory (5) that the developing retina is highly sensitive to any disruption of its oxygen supply, whether hyperoxemic or hypoxemic,

Received January 21, 1994; accepted July 5, 1994.

Correspondence: John S. Penn, Ph.D., Director, Arkansas Center for Eye Research, University of Arkansas for Medical Sciences/Slot 523, 4301 W. Markham St., Little Rock, AR 72205-7199.

Supported in part by a grant from the National Eye Institute (EY 07533-07), a development grant to the University of Arkansas for Medical Sciences from Research to Prevent Blindness, Inc. (RPB), the Dolly Green Scholars Award to J.S.P. from RPB, funds from the Arkansas Center for Eye Research Foundation, Alcoa Foundation, the Fraternal Order of Eagles, and Alcon Laboratories, Inc. warrants consideration. Recent studies in animals $(6,7)$ have addressed this issue.

Exposure to variable hyperoxia has been shown to be a much more effective stimulus of proliferative retinopathy in the newborn rat than exposure to constant hyperoxia (6). This previous study compared the effect of a cyclic variation of oxygen between 80 and $40 \%$ with that of a constant $80 \%$ exposure. An exposure paradigm that incorporates fluctuations in oxygen level is clearly more representative of the neonatal setting than the constant exposures typical of animal studies. Still, variations in inspired oxygen $\left(\mathrm{FiO}_{2}\right)$ between 80 and $40 \%$ do not accurately reffect the therapeutic levels received by premature infants. Neonates suffering apnea, patent ductus arteriosis, bronchopulmonary dysplasia, or one of a number of other respiratory complications often fluctuate between hyperoxic and hypoxic arterial partial pressures during therapy. This subpopulation of neonates is at high risk for $\operatorname{ROP}(8,9)$. To more closely model the clinical setting, we have now used an exposure cycle that varies 
between 50 and $10 \% \mathrm{FiO}_{2}$. Ambient hypoxia is necessary to produce episodes of low $\mathrm{PaO}_{2}$ in intrinsically healthy newborn rats.

\section{METHODS}

Upon birth, litters of Sprague-Dawley rats and their mothers were placed in a variable oxygen environment that consisted of $24 \mathrm{~h}$ of $50 \%$ oxygen followed by $24 \mathrm{~h}$ of $10 \%$ oxygen. The $10 \%$ oxygen atmosphere was produced by mixing appropriate fractions of pure nitrogen and room air. The oxygen level continued to alternate between 50 and $10 \%$ every $24 \mathrm{~h}$ for $14 \mathrm{~d}$, the time required for a room-air raised rat to complete retinal vascular formation. Simultaneously, rats were exposed to a similarly alternating exposure composed of 80 and $40 \%$ oxygen levels (Fig. 1). Other litters were raised in room-air conditions as a control. Some rats from each exposure group were killed on d 14 immediately after exposure to assess retinas for degree of avascularity. The remaining rats were removed to room air on $\mathrm{d} 14$ and were killed on d 18 to assess retinas for abnormal neovascularization. A total of 16 litters (169 rats) were used for the experiments. There was no mortality caused by the oxygen treatments, although preliminary trials showed that extending exposure to $10 \%$ oxygen environments beyond $24 \mathrm{~h}$ could be lethal. Treated rats were weighed upon removal from oxygen, as were room-air-raised rats at the same age. All animal experimentation was performed with the highest standards of humane care and conformed to the NIH Guide for the Care and Use of Laboratory Animals.

Retinal avascularity. The degree of retinal avascularity was calculated for 21 rats $n=15$ and $n=6$ for $50 / 10 \%$ and $80 / 40 \%$ treatments, respectively) using an adaptation of the method of D'Amato and Smith (10). This entailed

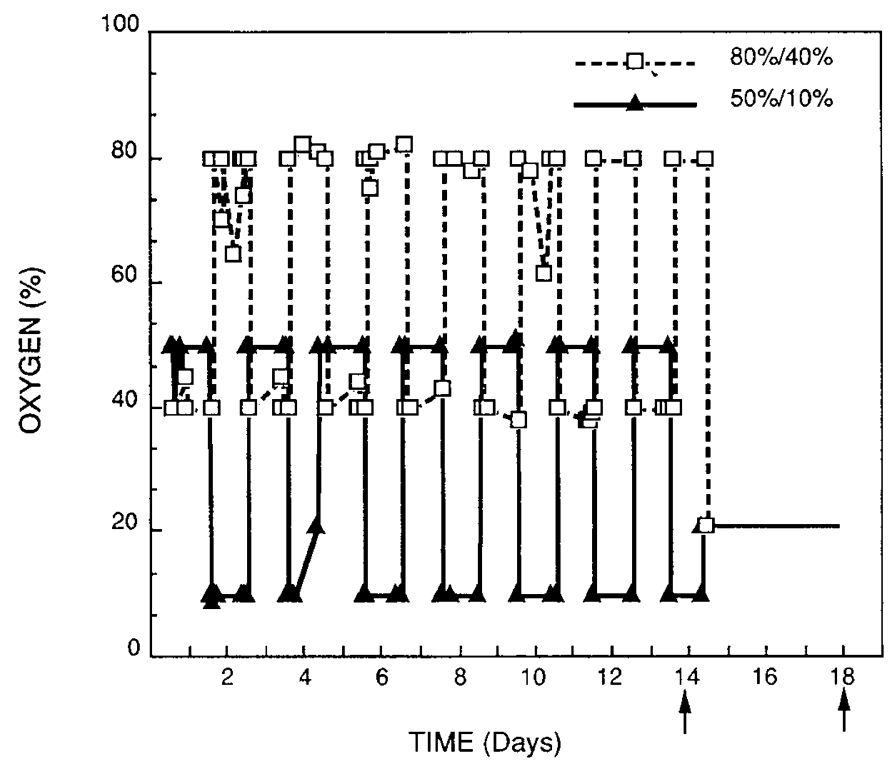

Figure 1. Oxygen exposure paradigm. This schematic is a real-time plot of the course of two typical oxygen exposures. The litters are placed in incubators within $4 \mathrm{~h}$ after birth and the oxygen level is adjusted every $24 \mathrm{~h}$ thereafter. The arrows denote times of killing. deeply anesthetizing the ratling with $40 \mathrm{mg} / \mathrm{kg}$ sodium pentobarbital and injecting $9.0 \mathrm{mg}$ of fluorescein isothiocyanate-dextran (145000 molecular weight, Sigma Chemical Co., St. Louis, MO) in a $40-\mu$ L PBS vehicle into the left ventricle. After $80 \mathrm{~s}$, the ratlings were killed, the eyes enucleated, and the retinas dissected and flatmounted on microscope slides and assessed using an inverted fluorescence microscope (Olympus IMT-2, Olympus Optical Co., Tokyo, Japan). Analysis of avascular regions required the aid of a computer-image analysis apparatus described elsewhere (6). The diameter of major arteries and veins was measured $1.0 \mathrm{~mm}$ from the optic nerve head at $\times 1000$ magnification with the aid of an ocular micrometer.

Retinopathy. Abnormal neovascularization was assessed in 73 rats on d 18 using fresh retinal dissections to produce flat-mounted retinas that were stained for ADPase activity. This histochemical staining procedure is a previously described adaptation (6) of a method developed by Flower $e t$ al. (11). This method stains only retinal vascular endothelia and their stem cells in rats of this age (12). The presence of preretinal neovascularization was then determined by trained observers using adjustment of the plane of focus at high magnification. To grade severity of pathology, a clock face was superimposed on the retinal surface. Areas between the separated quadrants of the flattened retinas were omitted from the assessment. Counting the number of clock hours occupied by pathologic vessel growth, the established unit of clinical assessment of ROP, yielded a semiquantifiable measure of severity. Retinas were scored independently by three masked investigators and the three assessments averaged for each retina. Selected retinas were removed from the slides after staining and assessment and were processed for histologic sectioning to confirm the presence of preretinal neovascularization identified in flat mounts. Tissue was fixed in $2.5 \%$ glutaraldehyde, dehydrated in an ethanol series, and infiltrated and embedded in Embed 812 (Polysciences, Warrington, PA). Sections were $0.5 \mu \mathrm{m}$ in thickness and were stained with $1.0 \%$ toluidine blue.

A few rats were allowed to remain in room air for $14 \mathrm{~d}$ after $50 / 10 \%(n=5)$ or $80 / 40 \%$ exposures $(n=5)$ before killing. These rats were enucleated, and the whole eyes were processed for histology by the same means as those used for flat-mounted retinas.

Systemic oxygen. To establish the clinical relevance of the 50/10 variable oxygen exposure, arterial partial pressures of oxygen were measured in five rats at the end of the last $50 \%$ oxygen-exposure period (d 13) and in five rats at the end of the last 10\% oxygen-exposure period (d 14). Age-matched room-air rats $(n=4)$ were also assessed. Measurements were made from $300 \mu \mathrm{L}$ of blood taken from the left ventricle of deeply anesthetized animals using a heparin-flushed $0.5-\mathrm{mL}$ syringe with a $22-$ gauge needle. During the procedure, rats were ventilated using a Harvard Rodent Ventilator (Model 683, Harvard Apparatus, South Natick, MA) at a rate and volume matched to that observed before anesthesia for each 
Table 1. Effect of variable oxygen exposures*

\begin{tabular}{|c|c|c|}
\hline Parameter & $50 / 10 \%$ & $80 / 40 \%$ \\
\hline Peripheral avascularity $\dagger$ (\% retinal area) & $25.2 \pm 7.6(15)$ & $8.1 \pm 3.4 \ddagger(6)$ \\
\hline Central avascularity (\% retinal area) $^{\circ}$ & $4.2 \pm 2.1(15)$ & $14.1 \pm 3.2 \ddagger(6)$ \\
\hline Neovascularization incidence $\$(\%)$ & $97.3(36 / 37)$ & $72.2(26 / 36)$ \\
\hline Severity $§$ (average clock hours/retina) & $8.0 \pm 2.2(36)$ & $4.2 \pm 2.1 \neq(26)$ \\
\hline \multicolumn{3}{|c|}{$\begin{array}{l}\text { * Values are mean } \pm \mathrm{SD} \text {. Sample sizes are in parentheses. } \\
\dagger \text { Assessment was made immediately after } 14-\mathrm{d} \text { oxygen exposure. } \\
\text { ‡ Significantly different from corresponding } 50 / 10 \% \text { value }(p \leq 0.005) \text {. } \\
\S \text { Assessment of severity and incidence was made after } 14-\mathrm{d} \text { oxygen } \\
\text { xposure and } 4 \mathrm{~d} \text { postexposure in room air. }\end{array}$} \\
\hline
\end{tabular}

treatment group (60 to 75 pulses per minute and 0.25 to $0.4 \mathrm{cc}$ ) and using the ambient oxygen level for each group $(10,50$, or $21 \%)$. Blood gases $\left(\mathrm{PaO}_{2}\right.$ and $\left.\mathrm{PaCO}_{2}\right)$ were analyzed using a Blood Gas Analyzer (Model 1306, Instrumentation Laboratory, Lexington, MA).

\section{RESULTS}

Body weights at $14 \mathrm{~d}$ were $24.3 \pm 2.8 \mathrm{~g}$ for room-airraised rats, $26.8 \pm 3.4 \mathrm{~g}$ for the $80 / 40 \%$ exposure group, and $19.8 \pm 2.3 \mathrm{~g}$ for the $50 / 10 \%$ group.

Retinal avascularity. Table 1 describes several parameters related to the pathology induced by the two variable oxygen exposure paradigms. The extent of avascular retinal area immediately upon removal from oxygen was $29.4 \%$ of the total retinal area in rats exposed to the $50 / 10 \%$ paradigm and $22.2 \%$ for rats in the $80 / 40 \%$ para$\operatorname{digm}(p \geq 0.05)$. The pattern of avascularity was different in the two groups: $50 / 10 \%$ rats displayed 3 times more avascular area in the peripheral retina than $80 / 40 \%$ rats (25.2 versus $8.1 \%$, respectively), but $80 / 40 \%$ rats had 3 times more avascular area in the central region (14.1 versus $4.2 \%$ ). Figure 2 illustrates the appearance of fluorescein-infused retinas from rats raised in room air and the two variable oxygen exposures. In addition to differences in the degree of retinal avascularity of the two exposure groups, there was also a substantial difference in vessel diameter as assessed with fluorescein infusion (Table 2). In 50/10\% rats, major retinal arteries and veins had an average diameter of 45.1 and $45.7 \mu \mathrm{m}$, respectively. In $80 / 40 \%$ rats, measurements averaged 27.8 and $35.3 \mu \mathrm{m}$, respectively $(p \leq 0.001$, for both artery and vein caliber).

Retinopathy. Four days after exposure, the incidence of abnormal retinal neovascularization in the two exposure groups was $97.3 \%$ for $50 / 10 \%$ rats, and $72.2 \%$ for $80 / 40 \%$ rats. The severity of neovascularization when it occurred was also greater in the 50/10\%-exposure group. Abnormal vessel growth was found in 8.0 average clock h of

Figure 2. Oxygen-induced retinal nonperfusion. These three panels illustrate the degree of retinal nonperfusion at the end of the 14-d exposure period and at the same time in room-air-raised controls. Vessels have been perfused with fluorescein-labeled dextran. Top panel, room air; middle panel, $80 / 40 \%$ oxygen exposure; bottom panel, $50 / 10 \%$ oxygen exposure. The increased caliber of major vessels and capillaries in the retinas of the 50/10\%-exposure group can be appreciated even at this low magnification.
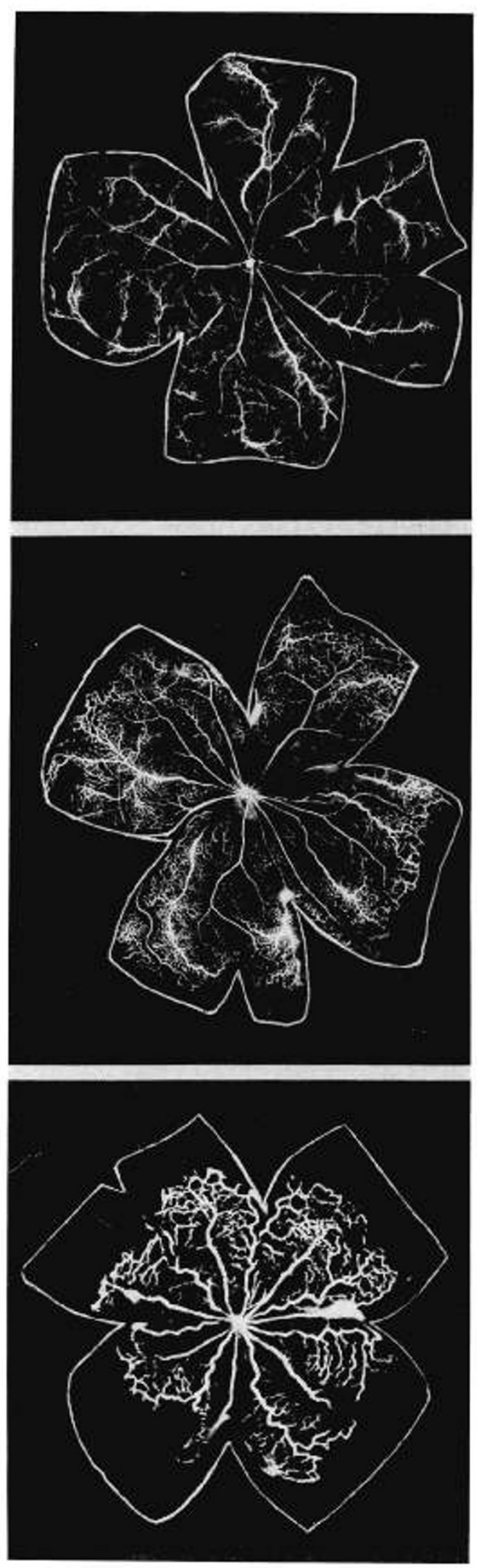
Table 2. Effect of variable oxygen exposure on vessel diameter

\begin{tabular}{lccc}
\hline & \multicolumn{3}{c}{ Vessel diameter } \\
\cline { 2 - 4 } & $50 / 10 \%$ & $80 / 40 \% \dagger$ & Room air $\dagger$ \\
\hline Major arteries & $45.1 \pm 6.5(13)$ & $27.8 \pm 5.3 \ddagger(12)$ & $28.7 \pm 3.6 \ddagger(11)$ \\
Major veins & $45.7 \pm 8.0(13)$ & $35.3 \pm 6.1 \ddagger(13)$ & $38.6 \pm 4.8 \ddagger(11)$ \\
\hline
\end{tabular}

$*$ Values are mean $\pm \mathrm{SD}$. Sample sizes are in parentheses.

$\dagger$ Significant difference between arteries and veins within exposure group $(p \leq 0.005)$.

$\ddagger$ Significantly different from $50 / 10 \%$ values $(p \leq 0.001)$.

retina in this group compared with 4.2 average clock $\mathrm{h}$ in the 80/40\% exposure group. Figure 3 illustrates ADPasestained retinas from the two oxygen exposure groups and from a room-air-raised rat of the same age.

Observation of retinas from the 50/10\% exposure group at higher magnification revealed preretinal vascular tufts that arose primarily at the peripheral-most extent of the major veins (arrow, Fig. $4 A$ ). Also evident were a high degree of tortuosity of the major arteries and frequent abnormal capillary buds at the peripheral extent of major arteries (arrows, Fig. 4B). In contrast, rats exposed to the $80 / 40 \%$ paradigm generally displayed narrow bands or ridges of preretinal vascular growth along the vascular/ avascular interface. These bands spanned the region between major arteries and veins.

Vitreal hemorrhages were seldom observed in the eyes of rats from the $80 / 40 \%$ exposure group (five of 42 , $12 \%$ ), but severe vitreal hemorrhages were found in nearly half of rats $(22$ of $52,42 \%$ ) exposed to the $50 / 10 \%$ paradigm. Figure $5 A$ depicts one such case in which the hemorrhage apparently emanated from preretinal vessels (thin black arrows, Fig. 5B, inset). The inner limiting membrane appeared relatively intact in this retinal location (thick black arrows, Fig. 5B, inset). There existed membranous extensions from the retinal surface that encompassed most, but not all, of the preretinal growth. Preretinal tufts like those often found in $80 / 40 \%$ rats were seen in these retinas (white on black arrow, Fig. $5 B$ ), but retinas from the $80 / 40 \%$ exposure group seldom displayed the membranous extensions. The retina in Figure $5 B$ was detached in the area immediately beneath the vitreal hemorrhage. This may have been due to the large dysplastic retinal fold underlying the hemorrhage or to tractional force provided by the membranous extensions and preretinal vessels. Because similar detachments were never seen in room-air rats, they are not believed to be artifactual, or if artifactual, the variable oxygen-exposed rats were much more susceptible to such artifact. A very limited sample of

Figure 3. Retinal neovascularization. These three panels illustrate the degree of abnormal vessel growth after a $4-d$ postexposure period in room air. Vessels are marked by ADPase activity. Top panel, roomair-raised rat shows no retinal pathology or avascular area at $18 \mathrm{~d}$ of age; middle panel, the $80 / 40 \%$ exposure group still has a small avascular zone in the retinal periphery which is bordered by abnormal capillary tufts (arrows); bottom panel, the 50/10\%-exposure group displays substantial peripheral avascularity and severe abnormal vessel proliferation, particularly at the peripheral extent of major veins (arrows).
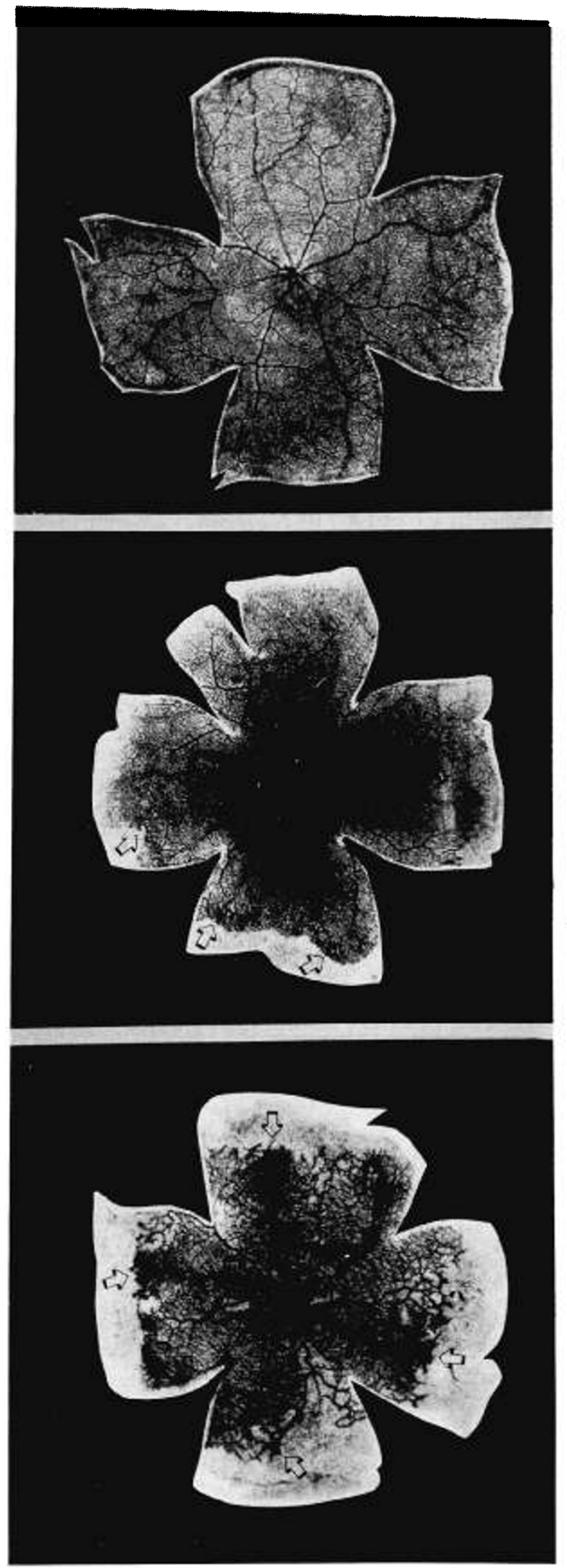

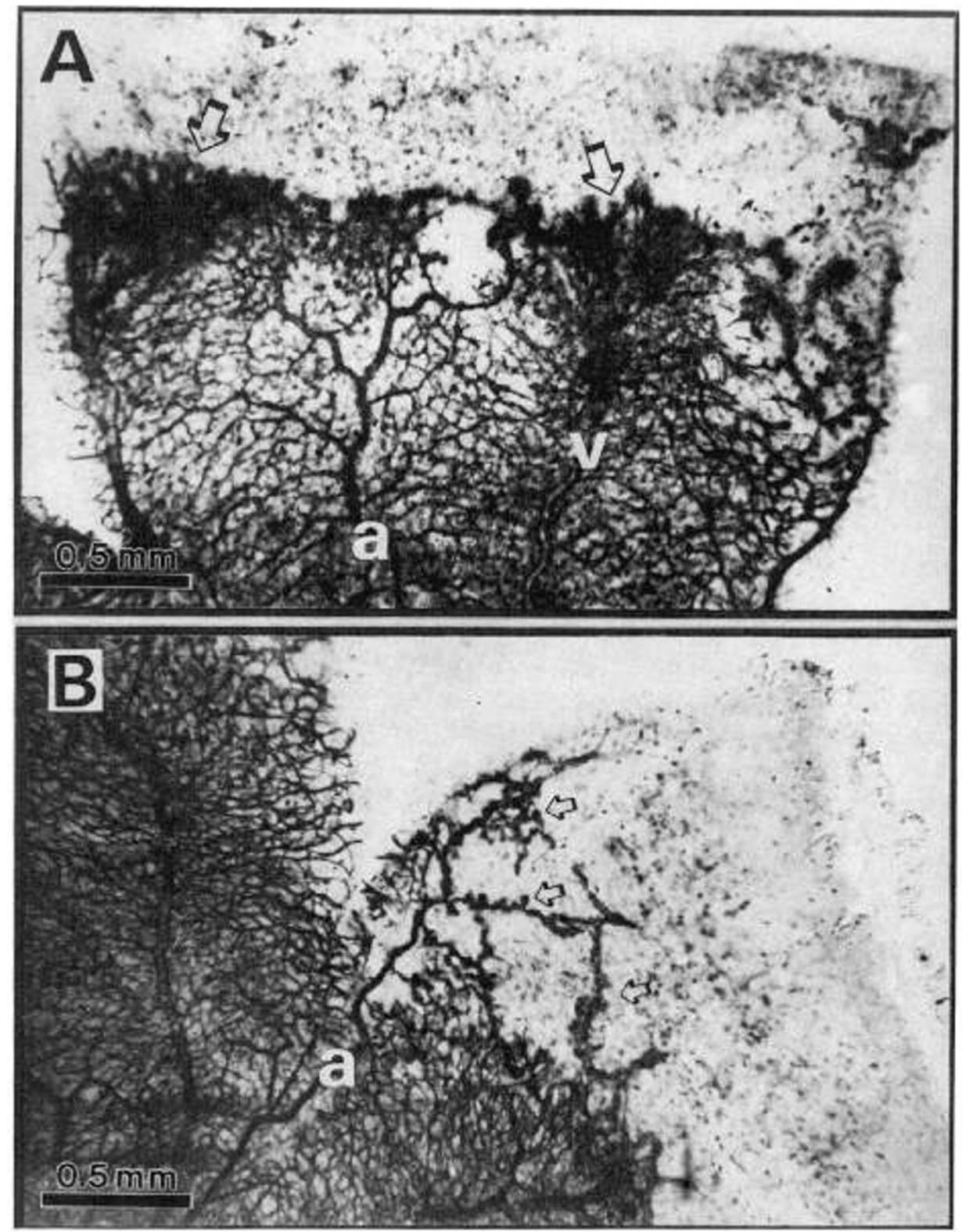

Figure 4. Vascular pathology in retinal flat-mount. At higher magnification, the abnormal vascular development of 50/10\% retinas is seen to manifest itself in at least two ways: Panel $A$, dense sheets of endothelial cells (arrows) overlay the peripheral extent of major veins, in many cases penetrating into the vitreous ( $a$, artery; $v$, vein); panel $B$, abnormal buds (arrows) extend from the peripheral extent of major arteries $(a$, artery). New capillaries in the superficial retina normally arise by differentiation of stem cells, rather than by endothelial budding.

$50 / 10 \%$ rats $(n=5)$ were maintained in room air for 14 d postexposure before killing. Two exhibited peripheral retinal detachment without vitreal hemorrhage. The other three appeared normal with the exception of sporadic dysplastic retinal folds like that illustrated in Figure $5 B$. The $80 / 40 \%$ exposure group yielded unremarkable retinas at this time.

Systemic oxygen. Results of the $\mathrm{PaO}_{2}$ assessment are shown in Table 3. Rats assessed at the end of the last period in $50 \% \mathrm{FiO}_{2}$ had an average $\mathrm{PaO}_{2}$ over 4 times higher than that of rats at the end of the last period in $10 \%$ $\mathrm{FiO}_{2}(\mathrm{kPa}=29.0$ versus 6.1 , respectively; 217.8 versus $45.8 \mathrm{~mm} \mathrm{Hg}$ ). Room-air-raised rats had an average $\mathrm{PaO}_{2}$ of $12.6 \mathrm{kPa}(94.8 \mathrm{~mm} \mathrm{Hg})$, which agrees well with previously published values (e.g. 13) and suggests that our apparatus functioned well for all other measurements. The $\mathrm{PaO}_{2}$ and $\mathrm{PaCO}_{2}$ values precisely agree with gasexchange theory for the 10 and $21 \%$ determinations, indicating that respiratory volume and frequency during blood collection were appropriately controlled. The 50\% determination yielded low $\mathrm{PaO}_{2}$ values, which may be explained by compromised pulmonary function caused by the extended exposure.

\section{DISCUSSION}

With respect to oxygen-induced retinopathy, the overall amount of oxygen that a subject receives is far less critical than other parameters of its administration. Raising rats in an environment cycling between 50 and 10\% 


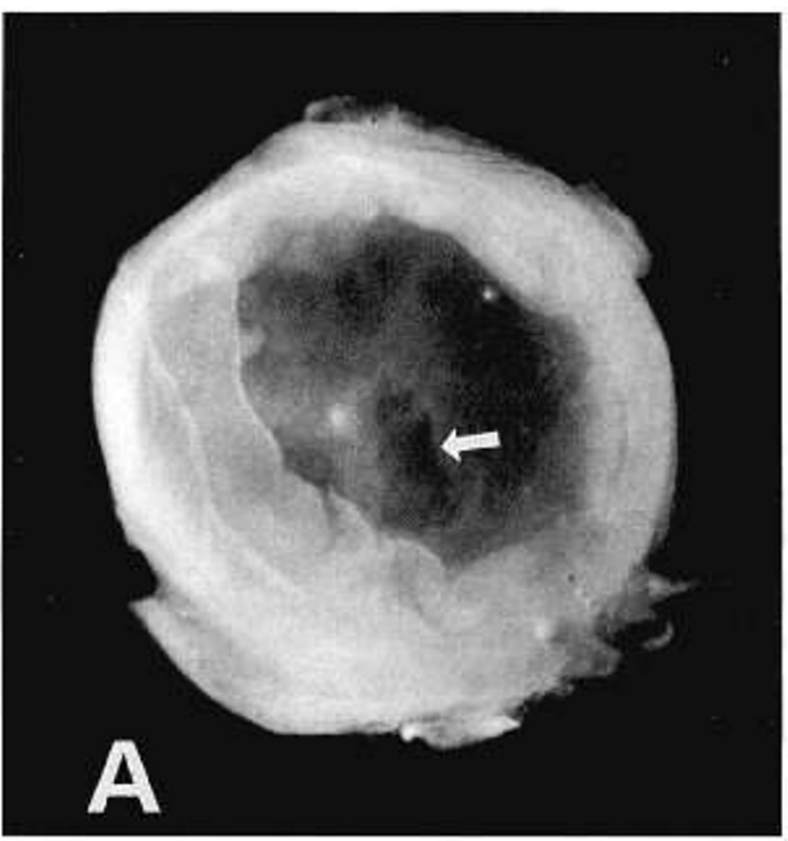

Figure 5. Vitreal hemorrhages and retinal detachments. Vitreal hemorrhages are a common result of the 50/10\% oxygen exposure. An "open sky" view shows a hemorrhage extending into the vitreous cavity with its origin near the posterior pole $(A$,arrow). A photographic montage of a transverse retinal section illustrates a similar hemorrhage near the retinal periphery $(B)$. Hemorrhages in this region often overlie retinal detachments. The preretinal tissue in this area consists of intact vessels (thin arrows, inset) and membranous extensions. The inner limiting membrane (thick arrows, inset) appears intact beneath the hemorrhage. Ridges of preretinal vessel growth also are seen in these retinas (white on black arrow), but they are less common here than in the $80 / 40 \%$ exposure group.

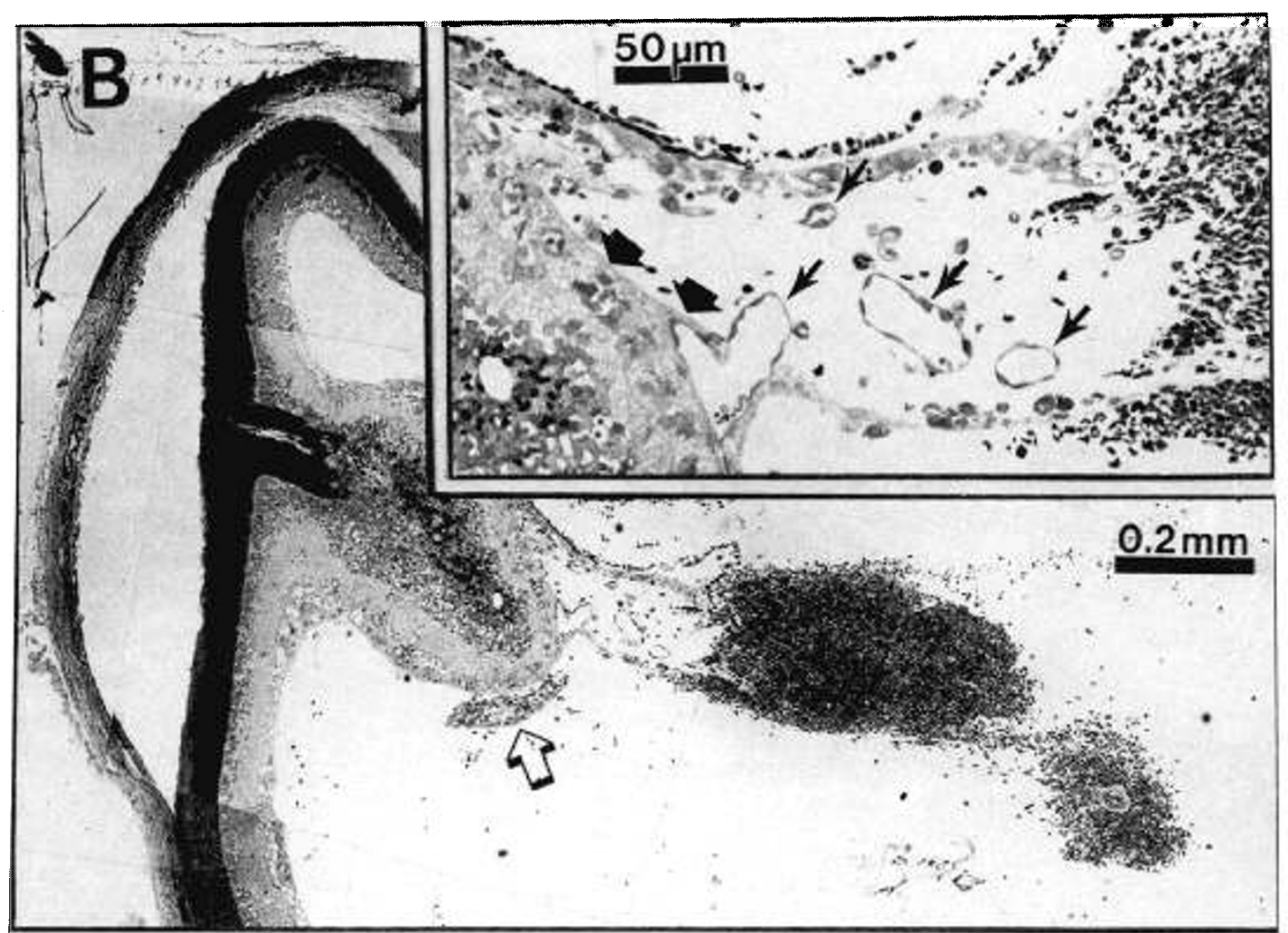

oxygen resulted in a much higher incidence and severity of retinopathy than did raising them in an $80 / 40 \%$ cycle, even though the latter received much more oxygen during the exposure period. This provides evidence that excessive oxygen alone is not the single overriding factor in retinopathy of prematurity and that episodal hypoxia may play a critical role in the pathogenesis.

Many studies have addressed the effect of local hypoxia on retinal structure and function (14-17) including experiments describing a stimulation of vascular endothelial mitosis in the rat retina (18). A recent study (19) suggests that vascular endothelial growth factor functions as a hypoxia-inducible angiogenic agent in some cell cultures and in tumors in situ. Furthermore, although inherently different by design, experiments conducted by Phelps and Rosenbaum (20) examined the effect of recovery in hypoxia after hyperoxic exposure of kittens. The authors found strikingly similar incidence of vitreal hemorrhage $(46 \%)$ as that found in the $50 / 10 \%$-exposure group in this study $(42 \%)$. Kittens that recovered in room air developed vitreal hemorrhages only $9 \%$ of the time. It should also be noted kittens exposed to hypoxia in this 
Table 3. Exposure effects on blood partial pressures*

\begin{tabular}{|c|c|c|c|c|}
\hline \multirow[b]{2}{*}{$\mathrm{FiO}_{2}(\%)$} & \multicolumn{2}{|c|}{$\mathrm{PaO}_{2}$} & \multicolumn{2}{|c|}{$\mathrm{PaCO}_{2}$} \\
\hline & $\mathrm{mm} \mathrm{Hg}$ & $\mathrm{kPa}$ & $\mathrm{mm} \mathrm{Hg}$ & $\mathrm{kPa}$ \\
\hline 50 & \multicolumn{2}{|c|}{$(n=5)$} & \multicolumn{2}{|c|}{$(n=5)$} \\
\hline 10 & \multicolumn{2}{|c|}{$\begin{array}{c}45.8 \pm 4.7 \\
(n=5)\end{array}$} & \multicolumn{2}{|c|}{$(n=5)$} \\
\hline 21 & $\begin{array}{r}94.8 \pm 14.5 \\
(n=\end{array}$ & 4) $12.6 \pm 1.9$ & $\begin{array}{r}42.2 \pm 4.1 \\
(n\end{array}$ & 4) $5.6 \pm 0.5$ \\
\hline
\end{tabular}

* Values are means \pm SD.

study showed poor weight gain, just as the $50 / 10 \%$ exposure caused in these experiments. Lastly, direct evidence of the clinical significance of hypoxia was presented by Katzman et al. (21), who found that more severe stages of ROP were closely associated with hours spent by infants with $\mathrm{PaO}_{2}$ below $50 \mathrm{~mm} \mathrm{Hg}$ and capillary $\mathrm{PO}_{2}$ below 35 $\mathrm{mm} \mathrm{Hg}$.

Just as important are several studies suggesting a relationship between fluctuation in retinal $\mathrm{Po}_{2}$ and $\mathrm{ROP}(22$ 25). The most rigorous of these is a study by Saito et al. (25) who showed a significant relationship between "hour-to-hour fluctuation" in arterial oxygen as measured by transcutaneous monitoring and progression of ROP. Of course, fluctuations in $\mathrm{PO}_{2}$ may simply be reflective of fragile infants who, because of other factors related to their condition, are at increased risk for ROP. The authors addressed this inherent complication by stringently limiting their study population and carefully assessing the infants' clinical conditions.

In comparing rats raised in the $80 / 40 \%$ cycle with those raised in the $50 / 10 \%$ cycle, another issue can be considered-namely, the relationship between the degree of retinal avascularity upon removal from the exposure chamber and the propensity for subsequent abnormal neovascularization. Several retinal conditions, including sickle cell retinopathy (26), diabetic retinopathy (27), and branch vein occlusion retinopathy (28), have in common a degree of retinal nonperfusion before abnormal vascular growth. This has led to the suggestion that the retinal vascular proliferation is the result of ischemia-induced hypoxia. It is not proven, however, that retinal nonperfusion leads to tissue hypoxia, and therefore a causal relationship between nonperfusion and vasoproliferation remains hypothetical for these pathologies.

Results of the present experiments support this relationship between the degree of retinal avascularity and subsequent abnormal proliferation. The 50/10\% cycle caused abnormal vessel growth in $97 \%$ of the rats, whereas the $80 / 40 \%$ exposure caused this pathology in $72 \%$ of the rats exposed in that manner. Also, the degree of pathology was 8.0 average clock $h$ of retina in the former group, but only 4.2 in the latter. The $50 / 10 \%$ rats had significantly $(p \leq 0.05)$ more avascular retinal area upon removal from the exposure chamber than did the $80 / 40 \%$ experimental group ( 29.4 versus $22.2 \%$ of total retinal area). If the stimulus for pathologic vascular proliferation is ischemia-induced hypoxia, the extent of which may well correlate to retinal avascularity, one would expect this relationship. Perhaps the extreme dilation of major vessels and capillaries in 50/10\% rats reflects an attempt to lessen the degree of retinal ischemia.

Interestingly, the pattern of retinal avascularity was different in the two exposure groups (Fig. 2). The vasoattenuation that characterizes oxygen-induced retinopathy in the rat is partly composed of two major capillary-free zones-one in the central retina surrounding the origin of vessels and one encompassing the retinal periphery (6). It has been proposed that the central avascular area is the result of obliteration of preexisting capillaries (29), and the peripheral avascular area is due to a slowing of the development of the retina's vascular network (30). Although we have no experimental evidence for either of these mechanisms, it is reasonable to assume that higher ambient oxygen would lead to higher tissue oxygen levels in the central retina where major retinal arteries are in close proximity. Diffusion of oxygen from these arteries may lead to death of existing vascular cells (vasoobliteration) by free-radical-mediated damage. Several studies have demonstrated biochemical evidence of such damage in retinas of oxygen-exposed animals (31-33). Conversely, a central retinal capillary-free zone might also be caused under these conditions by a cessation of capillary formation due to elevated tissue $\mathrm{Po}_{2}$ in combination with continued growth and peripheral extension of the retina. In either case, the $80 / 40 \%$ exposure would be expected to exert a greater effect on the central retina than the 50/10\% exposure, and it does, creating a 3-fold-larger central avascular area.

The peripheral avascular zone, on the other hand, is three times larger in the $50 / 10 \%$ exposure group. We must assume that diffusion of oxygen from the choroid vasculature and existing retinal vessels creates a higher $\mathrm{PO}_{2}$ in peripheral retinal tissue of $80 / 40 \%$ rats than $50 / 10 \%$ rats, so hyperoxia alone cannot explain the retardation of centrifugal vessel growth in the latter exposure group. It is possible that, although the relatively high $\mathrm{PO}_{2}$ in the peripheral retina of $80 / 40 \%$ rats is an inhibitor of vessel growth in that region, the hypoxic insult of $10 \% \mathrm{FiO}_{2}$ to the newly forming vessels and/or their precursors in the $50 / 10 \%$ paradigm is more damaging to the vasoformation process. This would explain the larger avascular area found in this region of the 50/10\% exposure group.

The site of abnormal neovascular growth is the midperipheral vascular/avascular boundary in rats as it is in humans. This is expected if one assumes that local retinal ischemia is the stimulus for this growth. The existing retinal vasculature must provide the origin of the new abnormal vessels, but the adjacent avascular retina would be the likely source of the growth stimulus. It is, therefore, logical that $50 / 10 \%$ rats suffer the more severe effects of the two groups, because the degree of peripheral retinal nonperfusion is much greater in $50 / 10 \%$ rats than in their $80 / 40 \%$ counterparts. 
Our blood-gas analysis of 50/10\% rats demonstrated $\mathrm{PaO}_{2}$ levels like those commonly found in sick infants monitored in neonatal ICU (25). Partial pressures as low as $5 \mathrm{kPa}(\sim 35 \mathrm{~mm} \mathrm{Hg})$ are frequently seen as the result of apnea or other respiratory complications. Pressures as high as 25 to $30 \mathrm{kPa}(\sim 185$ to $225 \mathrm{~mm} \mathrm{Hg})$ are periodically seen after therapeutic oxygen levels are increased to counter respiratory illness. It appears that the more we fashion our animal exposures to model the conditions experienced by sick neonates at risk for ROP, the more severe and consistent our pathologic results become.

Another possible explanation for the higher incidence and severity of retinopathy in the $50 / 10 \%$-treatment group is the ratio of the two oxygen levels. In the $50 / 10 \%$ group, the ratio of highest to lowest ambient oxygen level is 5 , and the systemic result is a $\mathrm{PaO}_{2}$ that varies by nearly 5 times. The $80 / 40 \%$ exposure constitutes an ambient oxygen ratio of only 2 . Because the range of $\mathrm{FiO}_{2}$ variation remains at $40 \%$, the absolute range of $\mathrm{PaO}_{2}$ that results from this treatment is probably similar to that caused by the $50 / 10 \%$ exposure. However, one would expect a 2-fold or less variation in $\mathrm{PaO}_{2}$ in the $80 / 40 \%$ treatment, which may constitute a less severe insult.

The high incidence $(97 \%)$ of proliferative retinopathy found in $50 / 10 \%$ rats is significant, not only because it implies a role of hypoxia in the pathogenesis, but because it constitutes a model in which potential therapies designed to inhibit this process can be reliably tested. It is reasonable to suggest that hypoxic episodes and fluctuations of $\mathrm{PaO}_{2}$ are critical factors in the etiology of ROP. Our findings suggest that avoidance of these two perturbations should be emphasized by neonatalogists.

Acknowledgments. The authors thank J. Bunch for processing the manuscript and $\mathrm{K}$. Conaway for technical assistance. Dr. T. Wall provided expertise in measuring blood gases.

\section{REFERENCES}

1. Ashton N, Ward B, Serpell C 1953 Role of oxygen in the genesis of retrolental fibroplasia: a preliminary report. Br J Ophthalmol 37:513-520

2. Patz A, Hoeds LE, De La Cruz E 1952 Studies on the effect of oxygen administration in retrolental fibroplasia. Am J Ophthalmol 35:1248-1252

3. Kinsey VE 1956 Retrolental fibroplasia: a cooperative study of retrolental fibroplasia and the use of oxygen. Arch Ophthalmol 56:481-543

4. Johns KJ, Johns JA, Feman SS, Dodd DA 1991 ROP in infants with cyanotic congenital heart disease. Am J Dis Child 145:200-203

5. Lucey JF, Dangman B 1984 A re-examination of the role of oxygen in retrolental fibroplasia. Pediatrics 73:82-96

6. Penn JS, Tolman BL, Lowery LA 1993 Variable oxygen exposure causes preretinal neovascularization in the newborn rat. Invest Ophthalmol Vis Sci 34:576-585
7. Penn JS, Tolman BL, Henry MM 1994 Oxygen-induced retinopathy in the rat: relationship of retinal non-perfusion to subsequent neovascularization. Invest Ophthalmol Vis Sci 35:3429-3435

8. Gunn TR Eastdown J, Outerbridge EW, Aranda JV 1980 Risk factors in retrolental fibroplasia. Pediatrics 65:1096-1100

9. Hammer ME, Mullen PW, Ferguson JG, Pai S, Cosby C, Jackson KL 1986 Logistic analysis of risk factors in acute retinopathy of prematurity. Am J Ophthalmol 102:1-6

10. D'Amato RD, Smith L 1993 Microscopic visualization of the retina by angiography with high-molecular-weight fluorescein-labeled dextrans in the mouse. Microvasc Res 46:135-142

11. Flower RW, McLeod DS, Lutty GA, Goldberg B, Wajer SD 1985 Postnatal retinal vascular development of the puppy. Invest Ophthalmol Vis Sci 26:957_ 968

12. Penn JS, Tolman BL, Bandyopadhyay A, Mauldin DV 1991 Retinal vasoformation in the rat as illustrated by adenosine diphosphatase staining. Invest Ophthaimol Vis Sci 32:1147

13. Hauptman JG, DeJong GK, Blasko KA, Chaudry IH 1989 Measurement of hepatocellular function, cardiac output, effective blood volume and oxygen saturation in rats. Am J Physiol 257:R439-R444

14. Ashton N, Henkind P 1965 Experimental occlusion of retinal arterioles. $\mathrm{Br} J$ Ophthalmol 49:225-234

15. Ernest JT, Krill AE 1971 The effect of hypoxia on visual function: psychophysical studies. Invest Ophthalmol 10:323-328

16. Linsenmeier RA, Steinberg RH 1986 Mechanisms of hypoxic effects on the cat DC electroretinogram. Invest Ophthalmol Vis Sci 27:1385-1394

17. Steinberg RH 1987 Monitoring communications between photoreceptors and pigment epithelial cells: effects of mild systemic hypoxia. Invest Ophthalmol Vis Sci 28:1888-1904

18. Stefansson E, Wilson CA, Schoen T, Kuwabara T 1988 Experimental isch emia induces cell mitosis in the adult rat retina. Invest Ophthalmol Vis Sci 29:1050-1055

19. Shweiki D, Hin A, Soffer D, Keshet E 1993 Vascular endothelial growth factor induced by hypoxia may mediate hypoxia-initiated angiogenesis. Nature 359:843-845

20. Phelps DL, Rosenbaum AL 1984 Effects of marginal hypoxemia on recovery from oxygen-induced retinopathy in the kitten model. Pediatrics 73:1-6

21. Katzman G, Satish M, Krishnan V 1987 Hypoxemia and retinopathy of prematurity. Pediatrics $80: 972$

22. Majima A 1976 Problems on retinopathy of prematurity: statistical analysis of factors related to occurrence and progression of retinopathy, and fundus appearances and ocular functions in prematurely born subjects. Nippon Ganka Gakkai Zasshi 80:1372-1419

23. Prendiville A, Schulenburg WE 1988 Clinical factors associated with retinopathy of prematurity. Arch Dis Child 63:522-527

24. Ruiz MPD, LeFever JA, Hakanson DO, Clark DA, Williams ML 1981 Early development of infants of birth weight less than 1000 grams with reference to mechanical ventilation in newborn period. Pediatrics 68:330-335

25. Saito Y, Omoto T, Cho Y, Hatsukawa Y, Fijimura M, Takeuchi T 1993 The progression of retinopathy of prematurity and fluctuation in blood gas tension. Graefes Arch Ophthalmol 231:151-156

26. Raichand M, Goldberg MF, Nagpal KC, Goldbaum MH, Asdouriam GK 1977 Evolution of neovascularization in sickle cell retinopathy. Arch Ophthalmol 95:1543-1552

27. Shimuzu K, Kobayashi Y, Muraoka K 1981 Midperipheral fundus involvement in diabetic retinopathy. Ophthalmology 88:601-612

28. Michels RG, Gass JDM 1974 The natural course of retinal branch vein obstruction. Trans Am Acad Ophthalmol Otolaryngol 73:166-177

29. Ashton N, Blach R 1961 Studies on developing retinal vessels. Effect of oxygen on the retinal vessels of the ratling. $\mathrm{Br} \mathrm{J}$ Ophthalmol 45:321-340

30. Ventresca MR, Gonder JR, Tanswell AK 1990 Oxygen-induced proliferative retinopathy in the newborn rat. Can J Ophthalmol 25:186-189

31. Penn JS, Thum LA, Naash MI 1992 Oxygen-induced retinopathy in the rat: vitamins $C$ and $E$ as potential therapies. Invest Ophthalmol Vis Sci 33:18361845

32. Bougle D, Vert P, Reichart E, Hartemann D, Heng EL 1982 Retinal superoxide dismutase activity in newborn kittens exposed to normobaric hyperoxia: effect of Vitamin E. Pediatr Res 16:400-402

33. Taki M, Majima A, Takada M 1982 Causal relationship of lipid peroxide with experimental retinopathy of prematurity: I. Morphological distribution of lipid peroxide in kitten retina after oxygen administration. Nagoya Med J 28:115-120 